\title{
Novel Closed-Cell Gas-Reaction Holder Allows Characterization of Behavior of Bimetallic Nanoparticles at Elevated Temperatures and Gas Pressures
}

\author{
Lawrence F. Allard ${ }^{1}$, James E. Wittig ${ }^{2}$, Miaofang Chi ${ }^{1}$, Karren L. More ${ }^{1}$, Wilbur C. Bigelow ${ }^{3}$, \\ John Damiano ${ }^{4}$ and David P. Nackashi ${ }^{4}$ \\ 1. Materials Science \& Technology Division, Oak Ridge National Laboratory, Oak Ridge, TN, USA \\ 2. Dept. of Electrical Engineering \& Computer Science, Vanderbilt University, Nashville, TN, USA \\ ${ }^{3 .}$ Dept. of Materials Science \& Engineering, University of Michigan, Ann Arbor, MI, USA \\ 4. Protochips, Inc., 606 Hutton St., Raleigh, NC, USA
}

Recently described [1,2] closed-cell gas-reaction specimen holders have been shown to permit studies of nanoparticulate materials at elevated temperatures and under selected gas compositions at pressures up to a full atmosphere, while permitting resolution of atomic columns in, e.g. an aberration-corrected electron microscope. An unresolved issue with heating holders used in electron microscopes (including conventional environmental TEMs), is the ability to control, and accurately determine, the exact temperature of the specimen during the gas reaction. In our work, MEMS-fabricated Aduro ${ }^{\mathrm{TM}}$ devices provided by Protochips, Inc. have traditionally been calibrated using infrared imaging methods while heating the devices in a chamber either under vacuum, or under chosen gas pressures. These calibrations showed a strong correlation between pressure and temperature at a given current, particularly in the regime below 100 Torr [1]. The Aduro device, when assembled into the gas-cell configuration, is the upper element of the cell, and the gas is confined by a thin $\mathrm{SiN}_{\mathrm{x}}$ lower window spaced a few microns from the heater element. In this configuration, the heater device sees the column vacuum on the outside, and gases at various pressures inside the cell. Early experiments (e.g. studies of ordering transformations in Fe-Pt nanoparticles (NPs)) were conducted under reducing conditions $\left(4 \% \mathrm{H}_{2} / \mathrm{Ar}\right)$, with the assumption that the temperature in a "static gas" configuration would equilibrate roughly between the vacuum calibration result and the "at-pressure" calibration result. At a pressure of 700 Torr, we observed rapid sintering of $\sim 5 \mathrm{~nm}$ NPs at a presumed temperature of $500^{\circ} \mathrm{C}$, and also rapid ordering (nearly complete ordering in many NPs after $5 \mathrm{~min}$ ) (Fig. 1a,b). In earlier vacuum heating experiments, however, we saw minimal sintering and no coalescence at $500^{\circ} \mathrm{C}$ under vacuum heating, and even after $18 \mathrm{~h}$, no ordered, single-domain particles were observed [3]. It was clear that a more reliable knowledge of the temperature behavior in the cell as a function of pressure and static vs flowing gas conditions was needed, so we made direct infrared camera measurements of a working gas-cell heater element. Figure 2 shows the gas-cell holder tip in the calibration chamber, with the heater element at a nominal temperature of $1000^{\circ} \mathrm{C}$ (i.e. glowing, arrow). Also shown is a corresponding digital readout from the IR camera that allows accurate determination of the temperature of the thin heater membrane as a function of heater current [1]. Figure 3a shows the calibration of an Aduro device in the cell, with the lower window broken out to simulate the standard calibration procedure for individual chips fully exposed in the test chamber. The left-most curve shows the behavior of the device in vacuum, and the remaining curves show the higher currents needed to heat the device to a given temperature as the gas pressure increases (to 300 Torr max for the right-most curve). In comparison, Fig. $3 \mathrm{~b}$ is the calibration set recorded with the gas cell fully intact, with gas introduced in a "static" condition into the cell interior (a volume of only $1.55 \mathrm{~mm}^{3}$ ), while the chamber was evacuated to the millitorr regime. In this configuration, the calibration curve, with vacuum in the cell, mimics the similar calibration in Fig. 3a, and as pressure is increased, a much lower current is needed to achieve a given temperature, relative to that needed with the device immersed in the gas. As an example, for a device temperature of $700^{\circ} \mathrm{C}$, a current of $4.5 \mathrm{~mA}$ was required in vacuum, and also with vacuum in the cell, whereas a current of $7 \mathrm{~mA}$ 
was needed for 300 Torr in the full gas calibration. A current of only $5 \mathrm{~mA}$ was required to reach $700^{\circ} \mathrm{C}$ when the cell interior was charged at 300 Torr. Thus this example shows why earlier experiments with static gas in the cell gave observations that suggested a higher temperature than was desired. It is therefore likely that the higher temperature than expected experienced by the FePt NPs better explains the behavior of the NPs for both sintering and ordering phenomena than any effect of gas composition, i.e. reducing gas conditions. We have conducted similar studies of phase separation phenomena observed during oxidation-reduction reaction cycles in Au-Pd core-shell NPs, and these will also be described. [4]

\section{References:}

[1] L F Allard, et al., Microsc. Microanal. 18 (2012), p. 656.

[2] J F Creemer, et al., Ultramicroscopy 108(9) (2008), p. 993.

[3] J E Wittig, et al., Microsc. Microanal. 18(Suppl 2) (2012), p. 304

[4] Research at the Oak Ridge National Laboratory High Temperature Materials Laboratory and ShaRE User Facility was supported by the Office of Energy Efficiency \& Renewable Energy, Vehicle Technologies Program, and the Scientific User Facilities Division, Office of Basic Energy Sciences, U.S. Department of Energy,

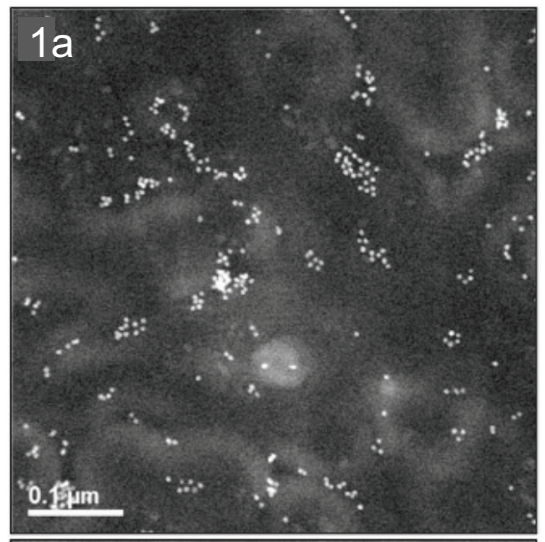

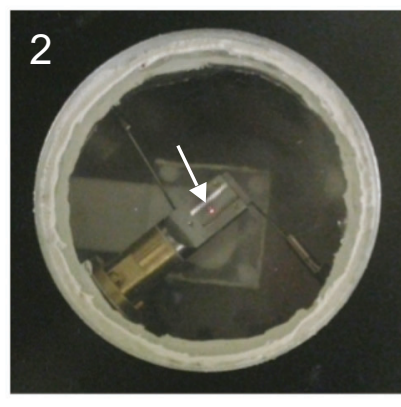

Arrow indicates heater element

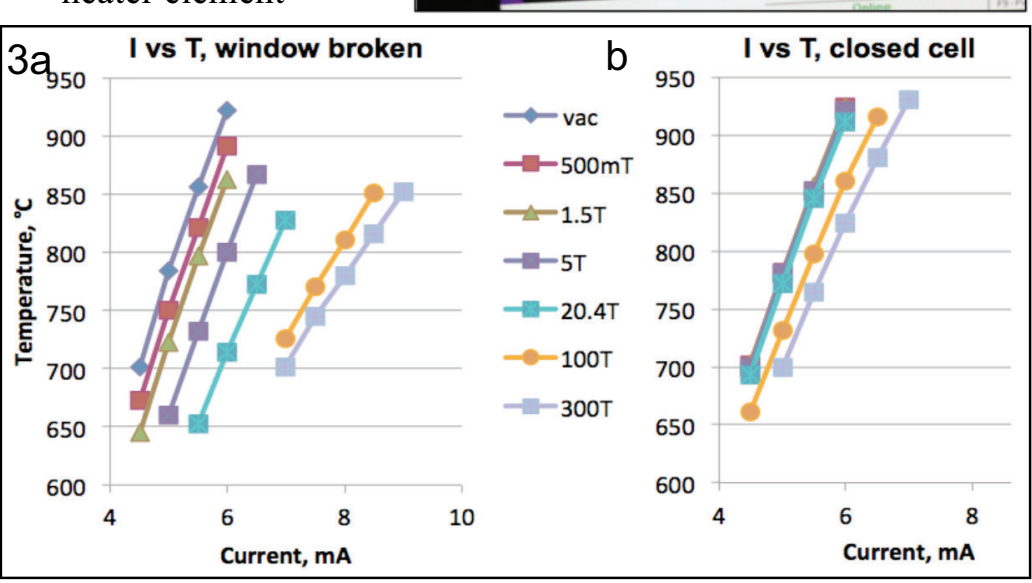

Figure 1. a) FePt nanoparticles prior to nominal $500^{\circ} \mathrm{C}-15 \mathrm{~s}$ anneal in 700 Torr $\mathrm{H}_{2} / \mathrm{Ar}$; b) after anneal, many nanoparticle groups show strong sintering and coalescence behavior; inset shows nearly complete single-domain ordering in FePt nanoparticle after total of 5 minutes anneal; see text for details.

Figure 2. Gas-cell tip in calibration chamber, at $\sim 1000^{\circ} \mathrm{C}$, with example infrared temperature readout. Figure 3. Current vs temperature calibrations, a) lower window broken to mimic standard in-chamber calibrations, and b) closed cell intact, showing much lower current (e.g. 300 Torr vs Vac) needed. 